\title{
Consistency on Multi-device Design
}

\author{
Rodrigo de Oliveira and Heloísa Vieira da Rocha \\ University of Campinas, Institute of Computing, Campinas SP 13081-970, Brazil \\ oliveira@ic.unicamp.br, heloisa@ic.unicamp.br
}

\begin{abstract}
We propose consistency priorities to support multi-device interface design minimizing the user's cognitive effort while performing the same task on different interfaces. The methodology is being evaluated through a framework that generates Pocket PC interfaces from desktop web pages. Initial results point to the acceptance of the approach.
\end{abstract}

Research sub-areas: design methods, mobile computing, web design.

\section{Introduction}

Mobile devices introduced a great challenge for Human Computer Interaction: to develop multi-device interfaces for today's applications. Some have tried device oriented designs with linear transformations, creating mobile interfaces from scratch, like Avantgo (www . avantgo . com) and Usable Net (www . usablenet . com); others looked for dynamic and automatic adaptations, but still focusing on the device [149]. These and other related approaches were well received, but the generated interfaces are different from the original in some aspects that complicate interaction with more than one device to perform the same task, especially when refinding and/or comparing information 810]. Many works addressed consistency and continuity problems focusing on user interface generation [5]6] and task migration [12], but their guidelines are generally not sufficiently concrete for an automatic interface framework. A recent proposal [1] solves the multi-device design problem by passing the control of every appliance to a handheld interface generated automatically. Despite the valued ideas, many device specific interaction types [13] important to each context of use can be lost on the process, besides the need to carry a mobile device to control everything.

We propose consistency priorities for multi-device interface design that aims to improve usability and the user's experience when performing similar tasks on different devices. Some prototypes were implemented for automatic desktop web page adaptation to handhelds, such as Pocket PCs and smartphones. Initial evaluations point to the acceptance of this approach. Formal user evaluations will be conducted to check these first impressions.

\section{Constructing the Proposal}

In order to highlight the problem and start a discussion over the main assumptions of this proposal, we are going to borrow an example from Sharp et al. [13] and adapt it to a multi-device context.

C. Baranauskas et al. (Eds.): INTERACT 2007, LNCS 4663, Part II, pp. 617-623, 2007.

(C) IFIP International Federation for Information Processing 2007 
Consider a person that had no contact with whatever interactive product to heat things. Now suppose we present him/her the stove with the task: "Heat this bottle of water the fast you can". The process of understanding how things work uses inductive inference based reasoning. Individuals draw conclusions about objects or events on the basis of previous observations of similar objects or events. On this example, the person has no experience with any related product, so the first minutes will be just for exploration. After a while, the individual will eventually understand how the stove works and accomplish the task, improving his/her mental model with information like "the more I turn this knob to the right, the more this flame becomes warm", "the more this flame becomes warm, the faster the water heats", etc. Now suppose we present him/her a thermostat and another task analogous to the previous: "Heat this room the fast you can". Both tasks are basically the same and so the individual will take the decision inductively, turning the thermostat knob most to the right. Although the applications had the same purpose (to heat things), their operation was inconsistent to the expected, thought the thermostat always heats the room in a constant speed, no matter how much the knob is turned to the right. The user could learn this new concept, but on a multi-device context with constant product refinement and maintenance, many usability attributes would be in risk, such as memorability, security, efficiency and others. Next subsection presents our proposal of consistency priorities to avoid ambiguous scenarios like this.

\subsection{Stating the Hypothesis}

Pyla et al. 12 argue that consistency needs to be better defined if it is to be the overriding factor in the design of multiple user interfaces. In fact, there isn't a consensus about what consistency really is and how it can be applied [1].

We think about consistency on the user's side. Individuals mentally draw conclusions about objects or events on the basis of previous observations of similar objects or events. These internal constructions that can be manipulated enabling predictions are called mental model [3]. Figure 1 sketches the user's mental model update cycle while executing tasks.

In order to help users form an accurate and useful mental model of a system while interacting with any of its interfaces, we suggest applying consistency on multi-device contexts using the following priorities:

1. Task Perception - the same control mechanisms to execute a task and their disposal on the interface. If these requirements cannot be followed with good usability on devices with different control attributes (e.g. size, weight, material, etc.) and properties (e.g. fluidity, flexibility, opacity, etc.), perception should be mapped to each device's interaction type maintaining usability.

2. Task Execution - the same actions flow to execute a task. If the control mechanisms available on a given interface had to be adapted for the others by the task perception priority, the actions flow should be maintained on a 


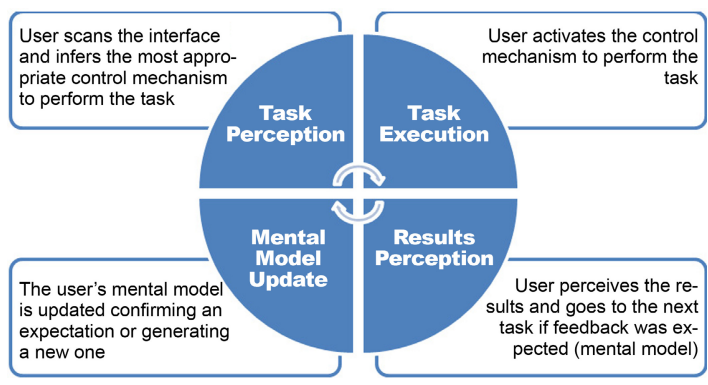

Fig. 1. User's mental model update cycle. Task perception and execution are the key processes to build a consistent mental model for decision making.

logical perspective. Although this may repass bad design decisions and lose opportunity to improve usability on each interface independently, user's decision making is supported under a consistent multi-device context, providing ease of learning/remembering and safety of use. Next priority improves efficiency.

3. Task Personalization - the ability to change task perception and execution according to the users' preferences. The goal is to achieve the best design for any user which is the configuration that user expects. This can be related to the personally consistent design concept [11, but with an active position for the user. As a result, efficiency and ease of expert use is provided to avoid the downsides of consistency [7].

It's important to understand the correct application of these consistency priorities as they can be easily misunderstood. For example, if an individual wants to check an account balance through an ATM machine, a tablet PC and a telephone, there is no possibility to perceive and perform the task in the same way. If the mentioned devices are important to the end user, adaptation to the contrasting interaction types is a price they are willing to pay. In fact, that's part of the task personalization priority. So the focus is to provide the same task perception and execution under a logical perspective, be it through words typed, written or said. This is in accordance with Nichols' work 11 about benefitting from user's experience, but opens space for the rich interaction types of the actual appliances in a consistent way.

\section{Towards an Empirical Validation}

\subsection{Proposing the Methodology}

On this section, we are going to take an application designed for multi-device access and improve it using our proposal. The application chosen is the Summary Thumbnail [8], a prototype designed to automatically adapt desktop web pages for handhelds. Here's how it works: the original web page is shrunken to fit 
horizontally on the smaller screen, text font is increased to improve legibility and letters are cropped from right to left until sentences fit on the available space. Complete texts can be read by accessing the detailed view through a click on the page, which moves to the original desktop interface with full scrolling. After applying the consistency priorities to Summary Thumbnail, we identified two issues:

1. Ambiguous task perception: the right-to-left cropping generates ambiguities, especially for navigational links (e.g. two links named "delete account" and "delete client" could be summarized to the same label "delete");

2. Different and ambiguous task execution: the new concepts of thumbnail view and detailed view along with their access procedures, resulting interaction fear (i.e. after clicking a link, there is no way to predict if it will visit it or move to the detailed view) and context loss on the original desktop interface with full scrolling.

The first problem could be solved by applying simple lexical analysis like the one given by Buyukkokten et al. 2, which will also maintain efficiency on runtime web page transformations. Additionally, we point a few restrictions:

- Long texts summarization: reducing long texts is time consuming and results tend to be questionable. The right-to-left cropping approach might be better;

- Navigational links summarization: anchors, buttons, hyperlinks and other access structures are the only interface objects to be considered for a more refined text summarization as their corresponding actions may be critical and shouldn't be misunderstood. Thought they are usually short sentences, the TF/IDF (term frequency / inverse document frequency) technique [2] could be enough when applied together with stem dictionaries. Additionally, it should be extended to use domain orientation. Different dictionary files could be generated remotely from database collections, each one containing information of term occurrences in each particular domain, and used by the interface adapter according to the web page being summarized.

The Summary Thumbnail's second problem is more complicated as the detailed view is a whole new concept that doesn't exist on the original task model but is fundamental to the approach. However, as it seems to demand a low learning curve to understand its operation, we decided to maintain it, but with a smaller cognitive effort on the transition to and from the thumbnail view. Next section explains how it was implemented on the first prototypes.

\subsection{Constructing and Evaluating the Prototypes}

On the first prototype generation (see Figure 2a), we used focus-plus-context to provide a faster detailed view over the thumbnail. Full texts and normal sized images are presented inside a hint window whenever users point to the corresponding object on the page. They can even confirm the full text to stay on page and this information is stored for future accesses (task personalization). 


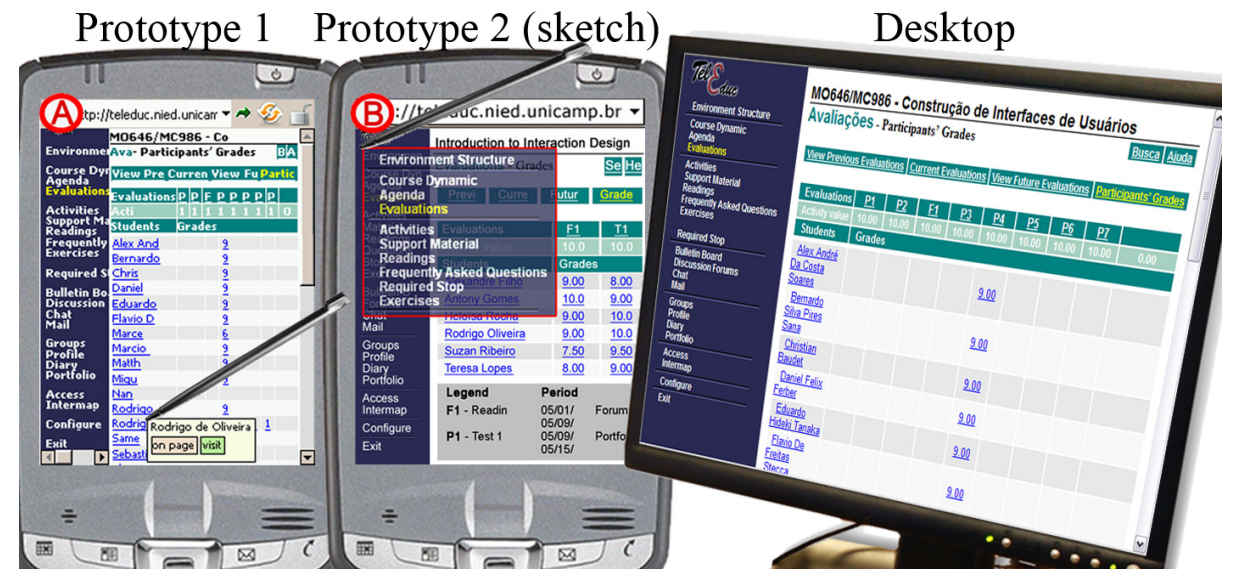

Fig. 2. Prototypes detailed view: (a) First generation: interface is shrunk, texts are summarized (right-to-left cropping) and fonts are increased. When the user points to summarized text, detailed view appears over the thumbnail without losing context (full text can be displayed on the thumbnail using the on page button and this information is stored for future accesses). If the user points to any link, an additional button is provided on the detailed view to visit it. (b) Second generation: TF/IDF [2] and stem dictionaries are used for better summarizations. The detailed view uses focus-pluscontext technique with a low opacity level to improve context view.
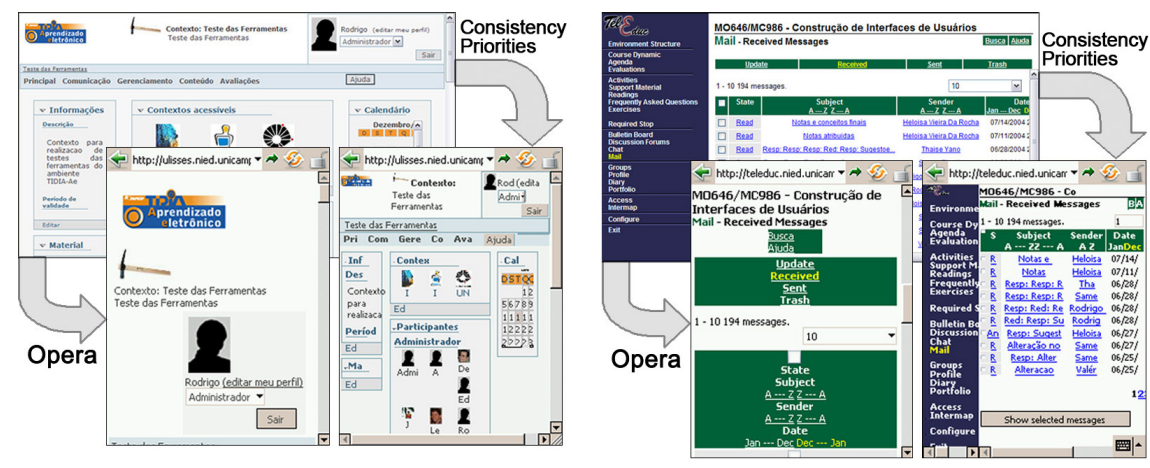

Fig. 3. Comparison between interfaces generated with the Consistency Priorities and Opera Fit to Screen. The first was much better evaluated on informal tests.

As the hint detailed view loses format attributes useful on iconic systems, we are developing the next prototype generation with the Direct Migration [10] (no transformation applied to the page) inside the hint window with a lower opacity value to improve context view (see Figure $2 \mathrm{~b}$ ).

Currently, the automatic interface adaptation doesn't require additional Internet traffic and takes less than two seconds to adapt a web page using the browser 
script interpreter. The hardware used was the HP iPAQ Pocket PC h2400 running Windows Mobile 2003 but could be any other with a CSS, DHTML and JavaScript compatible browser. Recently, the first generation prototype was informally tested on a few institutions with much better impressions than the awarded commercial solution by Opera (www . opera.com/products/mobile/ reviews). Figure 3 compares screens generated by both approaches.

\section{Conclusions}

The consistency priorities proposal aims to improve usability and the user's experience when performing similar tasks on different devices. The methodology is being tested through prototypes designed to automatically adapt desktop web interfaces for handheld screens. Informal evaluations revealed better impressions than a successful commercial approach. Next prototypes focus iconic interfaces and evaluations will be taken to verify the advantages of this proposal.

\section{References}

1. Berti, S., Correani, F., Mori, G., Paternó, F., Santoro, C.: Teresa: a transformationbased environment for designing and developing multi-device interfaces. In: Proc. CHI 2004 Extended Abstracts, Vienna, Austria, pp. 793-794 (April 2004)

2. Buyukkokten, O., Kaljuvee, O., Garcia-Molina, H., Paepcke, A., Winograd, T.: Efficient web browsing on handheld devices using page and form summarization. In: ACM Transactions on Information Systems, New York, USA, vol. 20, pp. 82-115 (2002)

3. Craik, K.J.W.: The nature of explanation. Cambridge University Press, Cambridge (1943)

4. Coninx, K., Luyten, K., Vandervelpen, C., Bergh, J.V.D., Creemers, B.: Dygimes: dynamically generating interfaces for mobile computing devices and embedded systems. In: Proc. MHCI 2003, Udine, Italy, pp. 256-270.

5. Denis, C., Karsenty, L.: Inter-usability of multi-device systems: A conceptual framework. In: Seffah, A., Javahery, H. (eds.) Multiple User Interfaces, pp. 373-385. John Wiley \& Sons, Chichester (2003)

6. Florins, M., Trevisan, D.G., Vanderdonckt, J.: The Continuity Property in Mixed Reality and Multiplatform Systems: A Comparative Study. In: CADUI 2004, Funchal, Portugal, pp. 323-334 (2004)

7. Grudin, J.: The case against user interface consistency. CACM 32(10), 1164-1173 (1989)

8. Lam, H., Baudisch, P.: Summary thumbnails: readable overviews for small screen web browsers. In: Proceedings of CHI 2005, Portland, OR, pp. 681-690 (2005)

9. Lin, J.: Using design patterns and layers to support the early-stage design and prototyping of cross-device user interfaces. Doctoral Thesis, p. 557. University of California, Berkeley, California (2005)

10. Mackay, B., Watters, C., Duffy, J.: Web page transformation when switching devices. In: Proc. of the Mobile HCI 2004, Glasgow, Scotland, pp. 228-239 (2004) 
11. Nichols, J.: Automatically generating high-quality user interfaces for appliances. Doctoral Thesis. Pittsburg, Pennsylvania: Carnegie Mellon University, p. 322 (2006)

12. Pyla, P., Tungare, M., Pérez-Quiñones, M.: Multiple User Interfaces: Why consistency is not everything, and seamless task migration is key. In: Proceedings of the CHI 2006 Workshop on The Many Faces of Consistency in Cross-Platform Design (2006)

13. Sharp, H., Rogers, Y., Preece, J.: Interaction design: beyond human-computer interaction, 2nd edn., p. 800. Wiley \& Sons, Chichester (2007) 